\title{
Effects of cold stress and starvation on the liver of yellow drum Nibea albiflora: histological alterations and transcriptomic analysis
}

\author{
Qihui Zhu ${ }^{1,2, \#}$, Hongbin Song ${ }^{1,2, \#, ~ Y a n ~ Z h a n g ~}{ }^{1,2}$, Ruiyi Chen ${ }^{1,2}$, Lu Tian ${ }^{1,2}$, \\ Dongdong $\mathrm{Xu}^{1,2, *}$
}

\begin{abstract}
${ }^{1}$ Marine Fishery Institute of Zhejiang Province, Key Lab of Mariculture and Enhancement of Zhejiang province, Zhoushan 316021, Zhejiang Province, PR China

${ }^{2}$ School of Fisheries, Zhejiang Ocean University, Zhoushan 316022, Zhejiang Province, PR China
\end{abstract}

\begin{abstract}
The yellow drum Nibea albiflora is a marine fish of great economic value in China. Despite efforts to improve yields, aquaculture of this species has been hindered by increases in winter-related mortalities associated with cold temperatures and associated natural fasting periods. To better understand the molecular mechanisms that regulate stress responses in yellow drum during periods of cold and starvation, the effect of these stresses on the liver was investigated by performing comparative analyses among fish subjected to different temperatures and feeding strategies. The experiment lasted for $22 \mathrm{~d}$ and involved 4 groups: one fed group (control) and one fasted group at $16^{\circ} \mathrm{C}$, and one fed group and one fasted group at $8^{\circ} \mathrm{C}$. Our results showed that all stress-treated groups exhibited body weight loss during the experiment, demonstrating that both cold stress and fasting caused growth inhibition, but only the fish in the fasted group at $16^{\circ} \mathrm{C}$ showed a loss in the liver/body ratio, suggesting that starvation can cause mass loss in the liver while cold stress can result in mass loss in both liver and other tissues. Histological alterations were observed in the liver cells from stress-treated groups, also indicating mass loss in the liver during cold stress and starvation. Transcriptomic analysis showed that genes related to the metabolism of carbohydrates, lipids and amino acids were the most enriched differentially expressed genes during the challenge conditions. These findings can help reveal molecular mechanisms regulating the stress responses of yellow drum exposed to cold and starvation.
\end{abstract}

KEY WORDS: RNA-seq $\cdot$ Cold stress $\cdot$ Starvation stress $\cdot$ Histological alterations $\cdot$ Yellow drum

\section{INTRODUCTION}

Winter mortalities (those associated with low temperatures) have been reported in a large number of fish species and have resulted in severe economic losses, especially in regions and countries that are dependent on aquaculture (Kirjasniemi \& Valtonen 1997, Hurst 2007, Ibarz et al. 2010, Zhang et al. 2019). This problem is especially critical for species cultured in net cages or ponds, where water temperatures are easily affected by weather and fish are

*Corresponding author: xudong0580@163.com

\# These authors contributed equally to this work unable to escape to warmer waters. When the temperature drops, growth, nourishment and vital activities are minimised, causing a natural fasting period. Although extreme cold shocks can be the direct cause of mortality in some cases, cold stress and the associated induced starvation are commonly known to drive most overwinter mortalities in farmed fishes (Hurst 2007, Donaldson et al. 2008). Previous work has demonstrated that starvation can decrease glucose and triglyceride levels, increase serum cholesterol and reduce glycogen and total lipid concentra-

(C) The authors 2020. Open Access under Creative Commons by Attribution Licence. Use, distribution and reproduction are unrestricted. Authors and original publication must be credited. 
tions in the fish liver (Favero et al. 2020); temperature drops in fish can induce a cascade of events, including cold-induced fasting, thermal stress, metabolic depression, immune suppression and infections by opportunistic pathogens (Hurst 2007, Donaldson et al. 2008). However, the molecular mechanisms underlying conditions of cold and starvation stresses, which drive the overwinter mortalities, are still poorly understood.

Though previous studies on fish responses to cold stress have mainly focused on physiological and biochemical aspects, efforts have also been made at the molecular level (Gallardo et al. 2003, Hyvarinen et al. 2004, Xu et al. 2015, Wen et al. 2018). With the advancement of transcriptome sequencing, RNA-seq has emerged as a powerful tool to study the molecular responses of fish relative to environmental changes. For example, Si et al. (2018) conducted RNA-seq analysis on half-smooth tongue sole Cynoglossus semilaevis and showed that metabolic pathways, especially lipid metabolism, are critical for salinity adaptation. Huth \& Place (2013) also generated a reference transcriptome of emerald rockfish Trematomus bernacchii gill tissue and found that many genes associated with metabolism were up-regulated in response to cold stress. A transcriptomic study on the large yellow croaker Larimichthys crocea showed that pathways involved in type I fatty acid synthesis, $\beta$-oxidation, polyunsaturated fatty acid synthesis, oxidative phosphorylation and molecular chaperones may take part in cold resistance (Zhang et al. 2019). Bilyk \& Cheng (2013) studied the transcriptome of the bald notothen Pagothenia borchgrevinki and found that genes associated with ubiquitin-protein ligase activity, protein ubiquitination and protein binding may play essential roles in cold temperature functioning.

The yellow drum Nibea albiflora is a sciaenid fish endemic to the subtropical and temperate coastal waters of the Northwest Pacific, ranging from the South China Sea to the coastal waters of Japan and Korea. Sea-cage farming of this fish has rapidly spread throughout the coastal regions of southeast China; however, yellow drum farms have frequently experienced mass mortalities during the overwinter stages, resulting in substantial economic loss to the aquaculture industry. When water temperature drops below $12^{\circ} \mathrm{C}$, yellow drum become minimally active and exhibit reduced food intake and slowed metabolism; their lethal temperature was reported to be $\sim 5^{\circ} \mathrm{C}$ (Xu et al. 2010). Furthermore, the overwinter period usually lasts 3-4 wk in the major production states (Zhejiang and Fujian Province). The cold tem- perature and induced fasting results in growth inhibition, histopathological changes, immune suppression as well as increased susceptibility to diseases which may lead to mass fish mortalities (Song et al. 2019). To date, no study has reported the effect of cold stress and starvation on the liver of yellow drum at the transcriptomic level.

In the present study, the effect of cold stress and starvation on the yellow drum liver was investigated via a comparative analysis at histological and transcriptomic levels, using fish subjected to different temperatures and feeding strategies. Results of this work will increase our knowledge of the molecular mechanisms associated with the response of yellow drum to low temperatures and starvation.

\section{MATERIALS AND METHODS}

\subsection{Experimental fish and treatment}

A total of 320 yellow drum individuals (95.37 \pm $15.30 \mathrm{~g}, 6 \mathrm{mo}$ old) were randomly collected from the hatchery at the research station of the Marine Fishery Institute of Zhejiang Province, Xixuan Island, Zhoushan, China. The fish were then randomly divided and placed into 8 fiber-reinforced plastic tanks (1000 l, 40 ind. $\operatorname{tank}^{-1}$ ), where they were acclimated (aerated seawater at $16.0 \pm 0.2^{\circ} \mathrm{C}, 27 \pm 0.1 \mathrm{ppt}$, natural sunlight photoperiod) for $2 \mathrm{wk}$ prior to the experiments. During acclimation, the aquarium water was exchanged twice a day, and seawater was maintained at $16^{\circ} \mathrm{C}$ by automatic temperature-controlled heaters (Xinlian). The fish were fed twice daily (at 8:00 and 16:00 h) with a commercial pelleted diet (Tianbang) to apparent satiation.

After acclimation, the fish were divided into 4 different treatment groups, and each treatment was duplicated. Two groups were maintained at $16^{\circ} \mathrm{C}$, but with different feeding strategies to investigate the effect of starvation: one group was routinely fed twice a day ('16fed', i.e. the control group) while the other group was not fed throughout the experiment ('16unfed' group). The other 2 groups were subjected to cold stress: the water temperature was decreased by $1^{\circ} \mathrm{C} \mathrm{d}^{-1}$ for $8 \mathrm{~d}$ until it reached $8^{\circ} \mathrm{C}$; and was maintained for $14 \mathrm{~d}$. One group was routinely fed until the fish stopped feeding at around $10^{\circ} \mathrm{C}$ ('8fed' group, i.e. cold-induced fasting, while the other group was not fed throughout the experiment ('8unfed' group). No mortalities were observed throughout the course of the experiments. At the end of the experiment (Day 22), 12 fish from each group were randomly sam- 
pled to record liver and body weight and body and total length, and 3 individuals from each group were randomly chosen for transcriptomic analysis. Fish were dissected immediately, and each liver sample was divided into 2 aliquots: one was immediately stored in liquid nitrogen for RNA isolation and the other was fixed with Bouin's solution for histological analysis.

\subsection{Histological observation}

Fixed liver samples were cut into $5 \mu \mathrm{m}$ thick sections with standard paraffin embedding methods and stained with haematoxylin-eosin. The stained samples were then observed under a microscope (Axio Imager A2; Zeiss) equipped with a digital camera (Axiocam 506; Zeiss).

\subsection{Total RNA extraction and Illumina sequencing}

The liver samples of 3 fish from each group (12 samples in total) that were stored in liquid nitrogen were prepared for transcriptomic analysis. Total RNA was isolated via a mirVana miRNA Isolation Kit (Ambion). An Agilent 2100 bioanalyzer (Agilent Technologies) was used to evaluate RNA integrity, and samples with RNA integrity number $\geq 7$ were subjected to subsequent analyses. The TruSeq Stranded mRNA LTSample Prep Kit (Illumina) was used to construct the libraries, and these libraries were sequenced on the Illumina HiSeq X Ten sequencing platform.

\subsection{Transcriptomic analysis and functional annotation}

Raw data were processed using Trimmomatic (Bolger et al. 2014). The reads containing ploy- $\mathrm{N}$ and the low-quality reads were removed to obtain clean reads. After removing the adaptor and low-quality sequences, the clean reads were subsequently aligned with Bowtie2 and mapped to the Nibea albiflora genome (National Center for Biotechnology Information [NCBI] accession: PRJNA577200, unpublished) via HISAT for gene annotation. Thus, gene functions were annotated using NCBI non-redundant, SwissProt and Clusters with the orthologous groups for the eukaryotic complete genomes databases. This was performed using BLASTx (Altschul et al. 1990) with a threshold E-value of $10^{-5}$. Based on the SwissProt annotation, gene ontology (GO) classification was con- ducted by mapping the relationship between SwissProt and the GO term. The genes were mapped to the Kyoto Encyclopedia of Genes and Genomes (KEGG) (Kanehisa et al. 2008) database to annotate the potential underlying metabolic pathways.

The FPKM (Trapnell et al. 2010) and read count value of each gene were calculated using Bowtie2 (Langmead \& Salzberg 2012) and eXpress (Roberts \& Pachter 2013). Correlation coefficients between samples were obtained to show the reliability of the experiment and rationality of sample selection. The DESeq functions 'estimateizeFactors' and 'nbinomTest' (Anders \& Huber 2013) were used to identify DEGs; p-values $<0.05$ and 'foldChange' values $>2$ or $<0.5$ were set as the threshold for significant differential expression. GO enrichment and KEGG pathway enrichment analyses of DEGs were respectively performed in $\mathrm{R}$ based on the hypergeometric distribution.

\subsection{Quantitative PCR (qPCR) validation}

Liver samples for transcriptomic analysis were also used for qPCR, and total RNA was extracted by the Trizol Reagent (Invitrogen) followed by a quality measurement via a $1.2 \%$ denaturing agarose gel. The concentration and quality of the RNA were measured by absorbance at $260 \mathrm{~nm}$ and agarose-gel electrophoresis, respectively.

The qPCR was performed on a quantitative thermal cycler (ABI StepOnePlus) using the SYBR Green real-time PCR mix (DRR041A; Takara), following a protocol described previously (Zhu et al. 2016). A dissociation step was completed after each PCR run to verify the specificity of the amplifications. For each sample, the qPCR was run in triplicate. $\beta$-actin was used as the reference gene according to previous studies in the yellow drum (Xu et al. 2018, Wang et al. 2019, Wu 2020). All primer pairs of the qPCR were designed by Primer Premier v.6.0; detailed information of the genes is provided in Table S1 in the Supplement at www.int-res.com/articles/suppl/ q012p359_supp.pdf. Relative gene expression levels were evaluated using the $2^{-\Delta \Delta C T}$ method (Livak \& Schmittgen 2001). The correlation coefficient was calculated using Microsoft Excel 2010.

\subsection{Statistical analyses}

All data are expressed as means \pm SE. A corrected Bartlett's test was used to check homogeneity of variances and found no significant difference in total 
variance for all data in this study. ANOVA and subsequent multiple comparison (Tukey) tests were conducted to compare significant differences among treatments using SPSS v.19.0 (IBM); p $<0.05$ was considered statistically significant.

\section{RESULTS}

\subsection{Effects of cold stress and starvation on growth performance of yellow drum}

At Day 0, total and body length showed no significant differences among groups. At Day 22, there was a significant increase in body weight in the $16 \mathrm{fed}$ group ( $p<0.05)$, while the other groups showed significant decreases ( $p<0.05$; Fig. 1). The body weight of the 16fed group was significantly higher $(p<0.05)$ than that of the other groups, while the fish in the other groups showed no significant difference between each other in body weight (Fig. 1). In terms of the liver/ body weight ratio, fish in the 16 unfed group had a significantly lower value $(\mathrm{p}<0.05)$, while no significant differences were observed in the other groups (Fig. 1).

\subsection{Histological alterations of the liver}

Under the microscope, liver cells of the $16 \mathrm{fed}$ control group appeared neat and orderly, with clear cell boundaries, a homogeneous cytoplasm and distinct
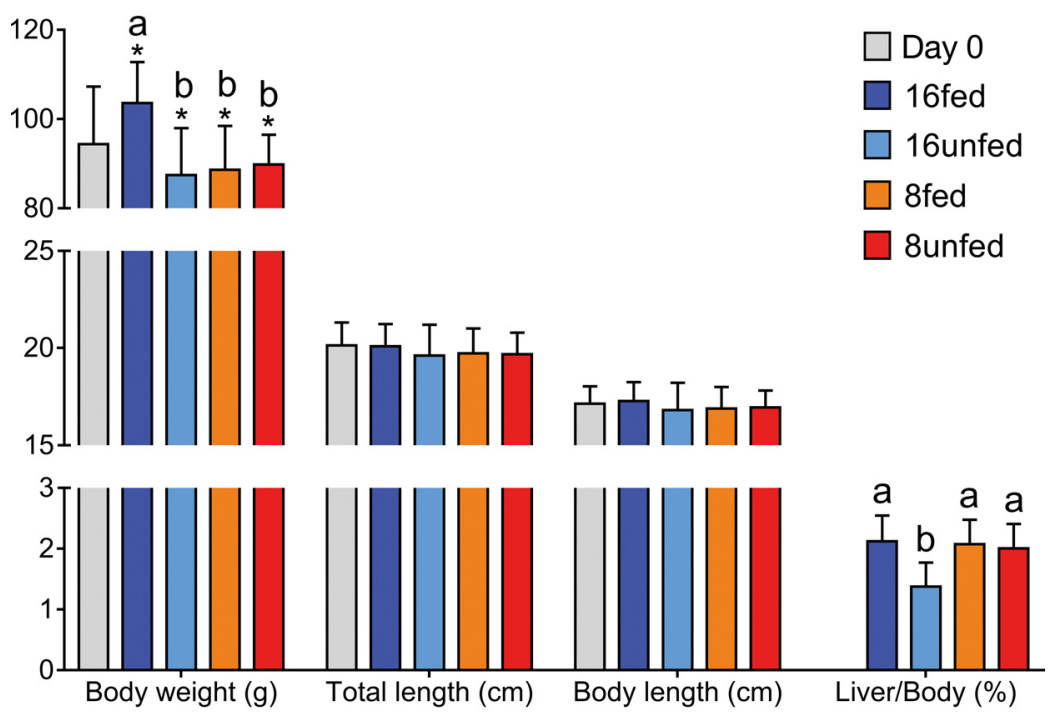

Fig. 1. Growth performance in different groups of yellow drum subjected to cold and starvation stresses. Data are shown as mean \pm SE $(n=12)$. (*) Significant differences between conditions before and after the experiments; different letters indicate significant differences between the treatment groups and the control group. Detailed data are listed in Table S2 well-rounded nuclei (Fig. 2A). The cells in the 16unfed group appeared slightly irregular in shape, with some displaying dispersion and vacuolisation (Fig. 2B). Noticeably, the cells from the low-temperature groups (8unfed and 8fed) showed obvious changes compared to those in the control group (Fig. 2C,D), with irregular cell shape, cytoplasm vacuolisation, blurred boundaries, atrophied and marginalised nuclei as well as cell dissolution.

\subsection{Illumina sequencing, reads mapping and differential expression analysis}

The raw data generated from the Illumina-based RNA sequencing of the 12 yellow drum samples were deposited into the NCBI database under accession number PRJNA589104. Detailed information of sequencing data and mapping is summarized in Table 1. The correlation coefficients between replicated samples were $>0.87$ (Fig. S1). More than $93 \%$ of high-quality reads had an average Phred quality score of $\geq 30$ at each base position, which were used for downstream analyses. Clean reads were mapped against the annotated genome of Nibea albiflora. As a result, over $94 \%$ of the clean reads from an individual sample could be successfully mapped, of which over $87 \%$ were uniquely mapped.

DEGs were analysed, and the DEG numbers that were obtained from all groups are displayed in Fig. 3. With 1221 up- and 1521 down-regulated DEGs, the $\square$ Day $0 \quad$ highest number of DEGs, followed by $\square$ 16fed the 8unfed-vs.-16fed group with 1163 up- and 1430 down-regulated DEGs. The 16unfed-vs.-16fed group contained 674 up- and 418 down-regulated DEGs, while the 8unfed-vs.-8fed group had only 38 up- and 20 down-regulated DEGs, which was the smallest number observed among the groups.

\subsection{Gene ontology and KEGG analysis}

In terms of the DEGs of the 8unfedvs.-16fed group, which represented the molecular response of the fish that suffered both cold and starvation stresses, GO annotation results (Fig. 4A) highlighted that GO terms relating to 'metabolic process' (236 

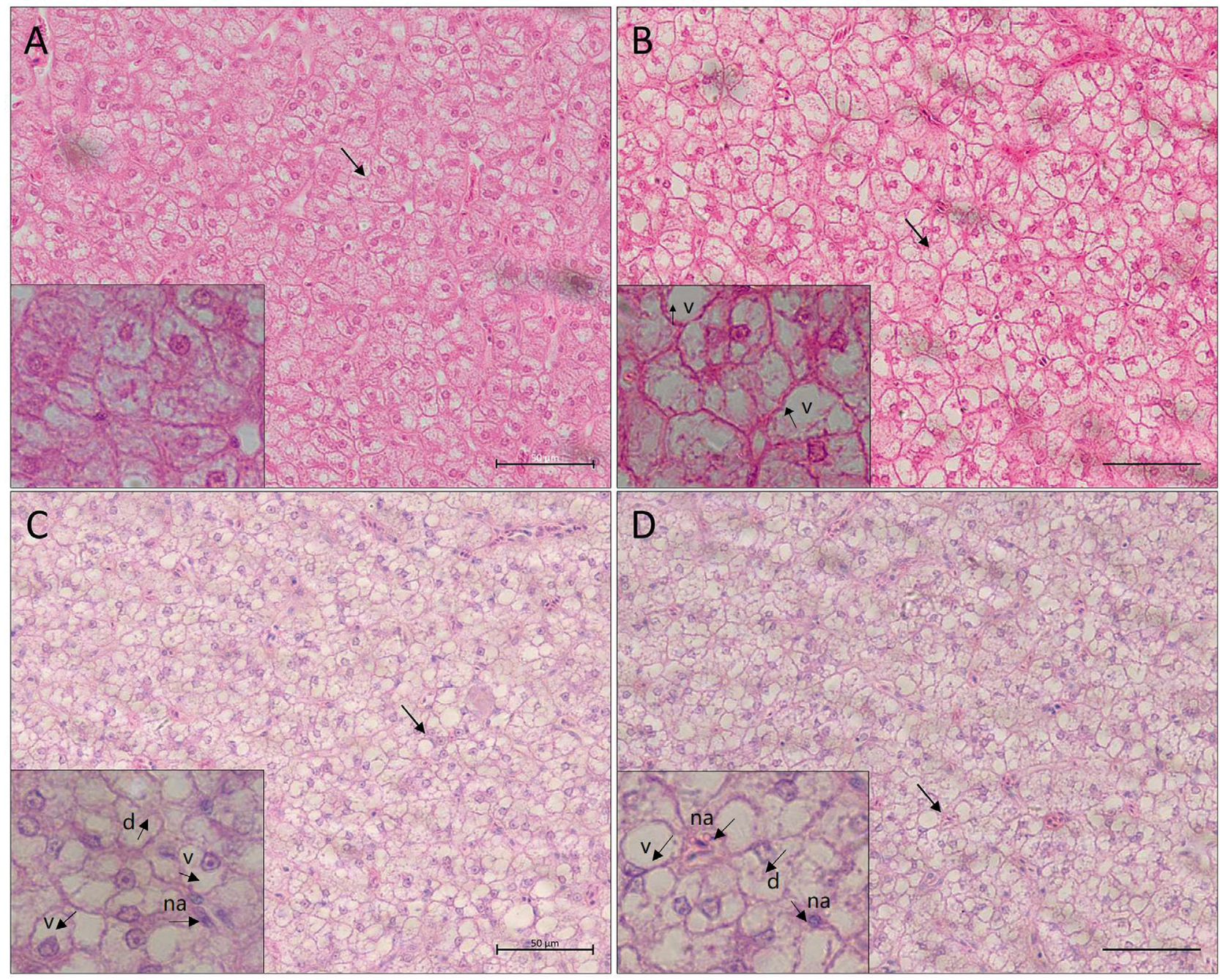

Fig. 2. Histological alterations in the livers of yellow drum subjected to cold and starvation stresses. Analyses were conducted on liver tissues sampled on Day 22 of the experiment from subjects from the (A) 16fed, (B) 16unfed, (C) 8unfed, and (C) 8fed groups. Scale bars: $50 \mu \mathrm{m}$. The lower-left corners are zoomed-in areas illustrated by the black arrows. Normal hepatocytes are shown in (A), and disordered arrangements of liver cells are shown in response to cold and starvation stresses, including vacuolisation, nuclear atrophy and even dissolution. Insets are zoomed-in areas indicated by the black arrows.

(v: vacuolisation; na: nuclear atrophy; d: dissolution)

genes) and 'cellular process' (210 genes) in the biological process category, as well as 'catalytic activity' (242 genes) and 'binding' (330 genes) in the molecular function category contained the highest number of DEGs. DEGs of the 8fed-vs. -16fed group showed the molecular response of the fish under cold stress and partial starvation stress, DEGs of the 16unfed-vs.-16fed group showed that of only starvation stress, and DEGs of the 8unfed-vs.-16unfed group showed that of cold stress under starvation conditions. However, the 4 GO terms that contained the most DEGs in the 8unfed-vs.-16fed group (GO terms of metabolic process, cellular process, catalytic activity and bind) also had the highest numbers of DEGs in the 16unfed-vs.-16fed group (Fig. 4B), the 8fed-vs. -16fed group (Fig. S2a) and the 8unfed-vs.-16unfed group (Fig. S2b), though with different gene numbers in different groups. In addition, KEGG enrichment analysis showed that for the DEGs of the 8unfed-vs.-16fed group, 130 genes were related to energy or metabolic pathways, with 69 and 48 genes found in the signal transduction and immune system pathways, respectively (Fig. 5A). Fig. 5B shows the KEGG enrichment result of the DEGs in the 16unfed-vs.-16fed group, where 87, 28 and 28 genes were related to metabolism, signal transduction and the digestive system, respec- 
Table 1. Illumina sequencing and mapping statistics of the yellow drum liver transcriptome. M: million base pairs

\begin{tabular}{|lcccc|}
\hline Group & Sample & $\begin{array}{c}\text { Total clean } \\
\text { reads } \\
\text { (M) }\end{array}$ & $\begin{array}{c}\text { Total } \\
\text { mapping } \\
(\%)\end{array}$ & $\begin{array}{c}\text { Unique } \\
\text { mapping } \\
(\%)\end{array}$ \\
\hline 16fed & S1 & 47.91 & 94.56 & 86.54 \\
& S2 & 47.10 & 94.72 & 87.31 \\
& S3 & 47.78 & 94.98 & 86.72 \\
16unfed & S4 & 46.98 & 94.95 & 88.7 \\
& S5 & 46.74 & 95.06 & 88.68 \\
& S6 & 47.45 & 94.07 & 87.56 \\
& S7 & 46.88 & 94.97 & 88.79 \\
& S8 & 38.87 & 94.66 & 88.11 \\
& S9 & 47.27 & 94.71 & 88.49 \\
& S10 & 46.84 & 94.66 & 88.26 \\
Aved & S11 & 46.74 & 95.04 & 88.33 \\
& S12 & 45.03 & 94.38 & 86.01 \\
& & 46.3 & 94.73 & 87.79 \\
\hline
\end{tabular}

tively. In terms of the DEGs in the 8fed-vs.-16fed group, KEGG annotation result (Fig.S3a) showed that 120, 68 and 61 genes were involved in metabolism, signal transduction and immune system pathways, respectively. As for the 8unfed-vs. -16unfed group, pathways of signal transduction, immune system, transport and catabolism and cell growth and death enriched the highest number of DEGs. For the DEGs of the 8unfed-vs.-8fed group, only 18 genes had GO annotation and 13 genes

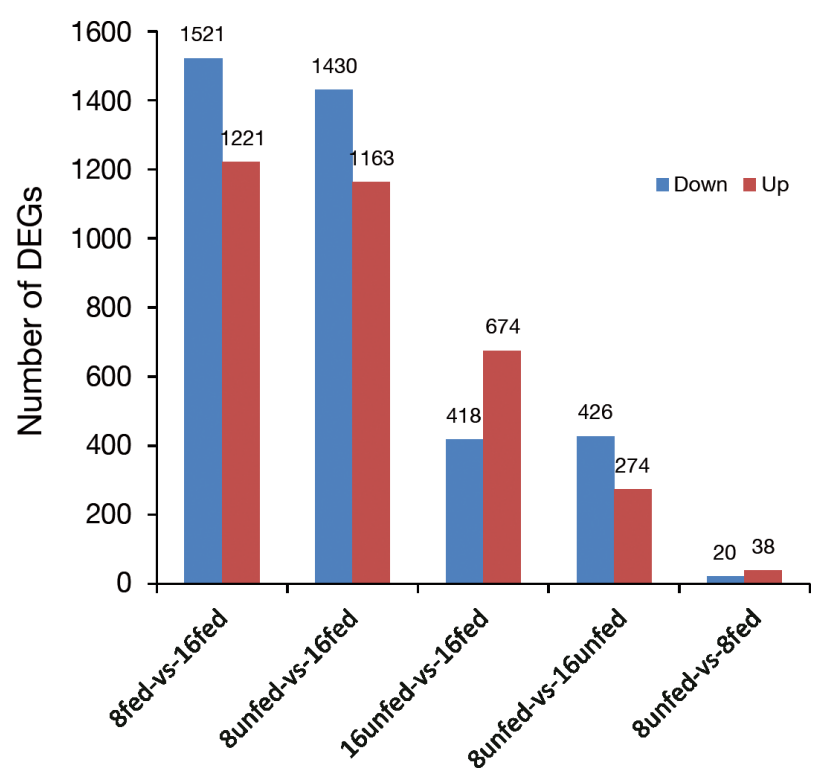

Fig. 3. Comparison of up-regulated and down-regulated differentially expressed gene (DEG) numbers between different groups of yellow drum subjected to cold and starvation stresses had KEGG annotation; 8 were related to protein or metal ion binding, and another 8 were related to lipid metabolism pathways.

The Venn diagram representing the DEGs in the 8unfed-vs.-16fed and the 16unfed-vs.-16fed group (Fig. S4) illustrated 420 DEGs that were specific to the 16unfed-vs.-16fed group, and 2070 DEGs specific to the 8unfed-vs.-16fed group, with 672 overlapping (i.e. these genes were DEGs in both groups). This result shows that 420 genes were regulated in expression under only starvation but not cold stress, 2070 genes under cold and starvation stresses combined and 672 genes in both of the treatments. We then set llog2Ratiol $\geq 2$ (fold change $\geq 4$ ) as a filtering threshold to identify the significant DEGs. As a result, for the DEGs specific to the 16unfed-vs.-16fed group, 10 significant DEGs were identified, and 6 of them were annotated as 3 metabolism-related genes, 1 signal transduction related gene, 1 digestive system gene and 1 endocrine system gene. In terms of the DEGs specific to the 8unfed-vs.-16fed group, 50 significant DEGs were identified, including 6 metabolism-related genes, 3 digestive system-related genes, 4 immune system-related genes, 2 endocrine system-related genes and 2 circulatory systemrelated genes. For the overlapping DEGs, 22 significant DEGs were obtained, 11 of which were enriched in metabolism-related pathways and 3 in the digestive system pathway. Specifically, 7 were involved in amino acid metabolic pathways, including the genes encoding peroxisomal sarcosine oxidase, methylmalonate-semialdehyde dehydrogenase, glycine cleavage H-protein, fumarylacetoacetase, proline dehydrogenase ( 2 genes) and the macrophage migration inhibitory factor. Detailed information of these significant DEGs is listed in Table S3.

\subsection{Validation of RNA-seq data by qPCR}

To validate the RNA-seq data, we randomly selected 8 DEGs for qPCR analysis, including NANS, LCE, IGLL5, PLIN2, GIMAP8, CHIA, AHSG and $L Y Z$, of which IGLL5, GIMAP8, CHIA and $L Y Z$ were immune-related, $L C E$ and PLIN2 were related to development and NANS and AHSG were associated with metabolism. The obtained Ct values were normalized against the reference gene $(\beta$-actin), and the fold change was calculated. As shown in Fig. 6, we obtained a correlation coefficient of 0.94 between the $\log _{2}$ (fold change) values of RNA-seq and the qPCR results, suggesting good consistency. 
A

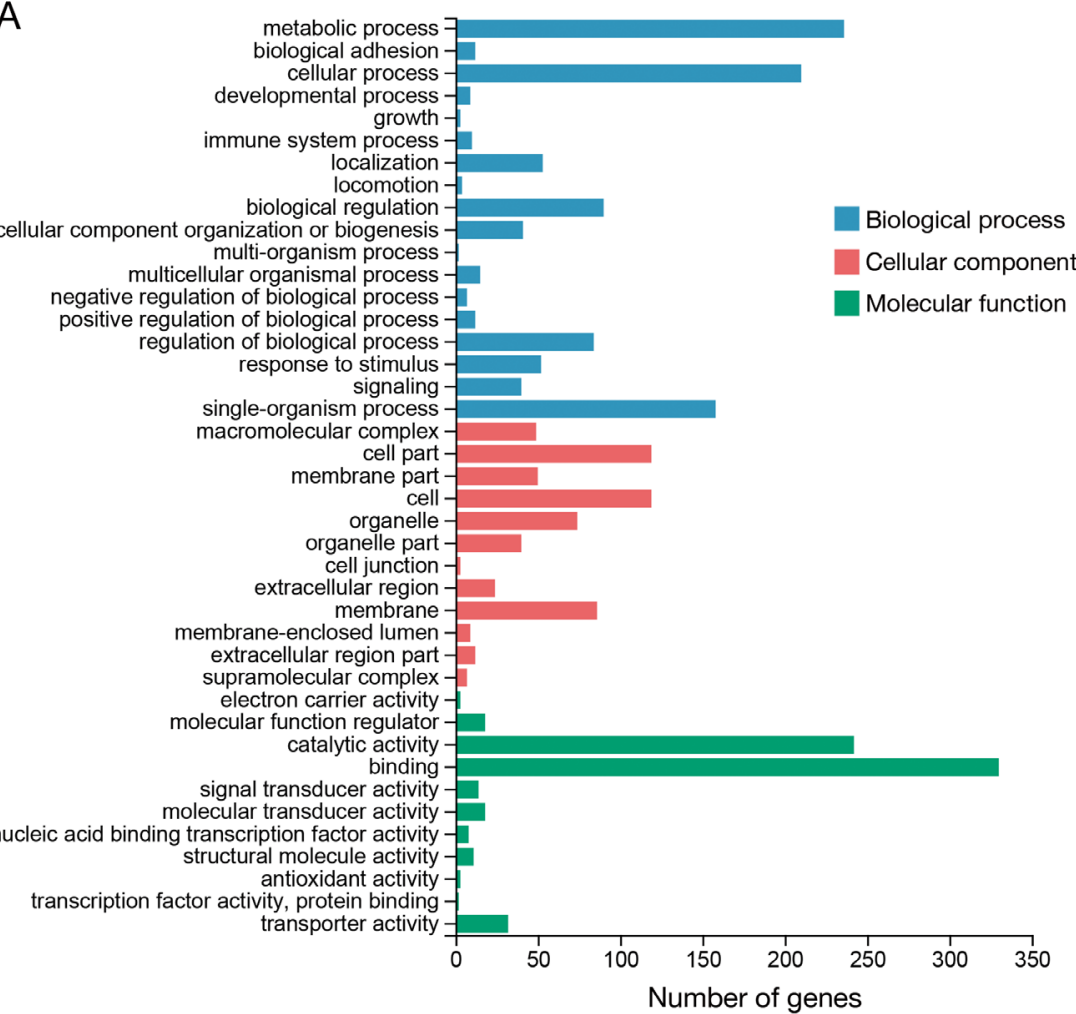

B

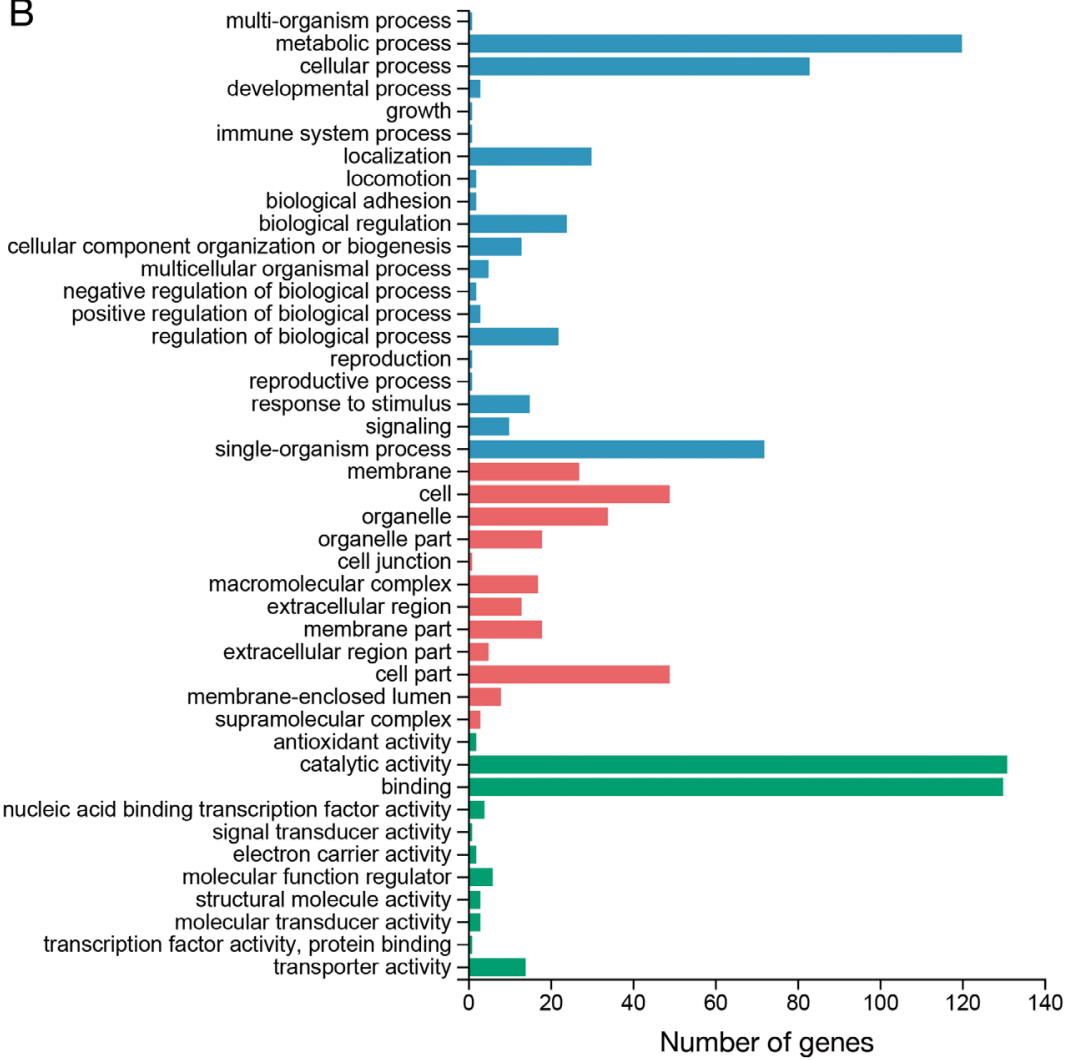

Fig. 4. Gene ontology (GO) terms based on the differentially expressed genes (DEGs) of the yellow drum (A) 8unfed-vs.-16fed and (B) 16unfed-vs.-16fed groups. Horizontal axis shows the number of DEGs enriched in each GO term

\section{DISCUSSION}

Dietary energy insufficiency along with conditions of low temperature can affect fish health and trigger mortality. This can cause great losses in fish production during winter. To better understand the effect of low temperature and starvation stresses on the liver of yellow drum, we conducted a comparative analysis among fish using different temperatures and feeding strategies.

We found that low temperature and starvation significantly affected the growth of yellow drum (Fig. 1), which is consistent with previous findings from studies conducted on black rockfish Sebastes melanops (Boehlert \& Yoklavich 1983) and sea bass Dicentrarchus labrax (Pastoureaud 1991). Body weight was significantly higher in the control group than in the groups subjected to starvation or cold stress. Cold-induced fasting was observed in the 8fed group, where food consumption gradually decreased along with decreasing temperature until the fish stopped ingesting food at $10^{\circ} \mathrm{C}$. As a result, for the 16unfed, 8fed and 8unfed groups, complete or partial starvation stress occurred, which is the main reason for the reductions in body weight. The liver is a large vital organ in fish which plays critical physiological roles including anabolism, catabolism, detoxication, the conversion of energy into proteins, lipids and carbohydrates, as well as the release of energy through glycogenolysis (Bruslé 1996). It has been reported in many animals that the liver can undergo a large reduction in mass during starvation as its constituent nutrients are mobilised (Thouzeau et al. 1999, Karasov et al. 2004, Lamosova et al. 2004). Because of a lack of glucose-6-phosphatase during starvation, most animals reduce and may completely deplete muscle tissue as well as hepatic glycogen stores. The glycogen stores would then be replenished through gluconeogenesis at the expense of lipids and pro- 
A

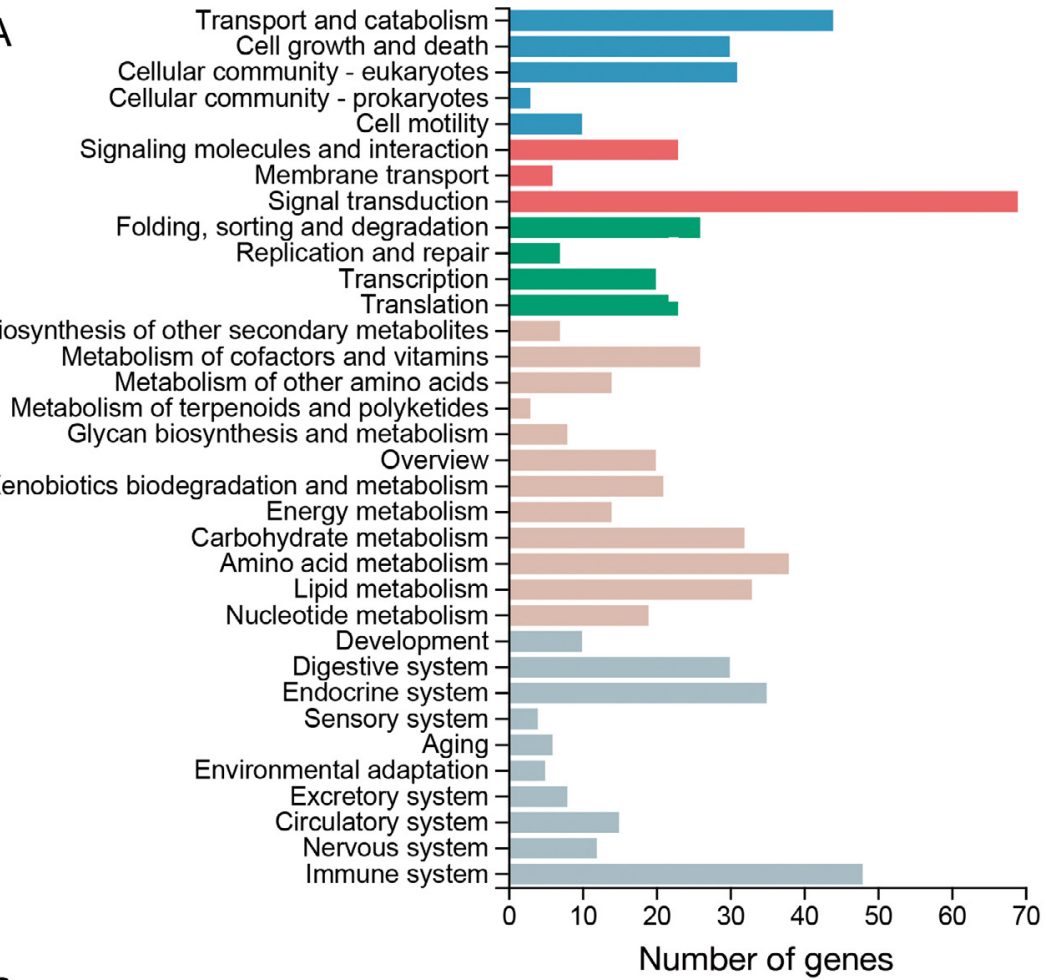

B

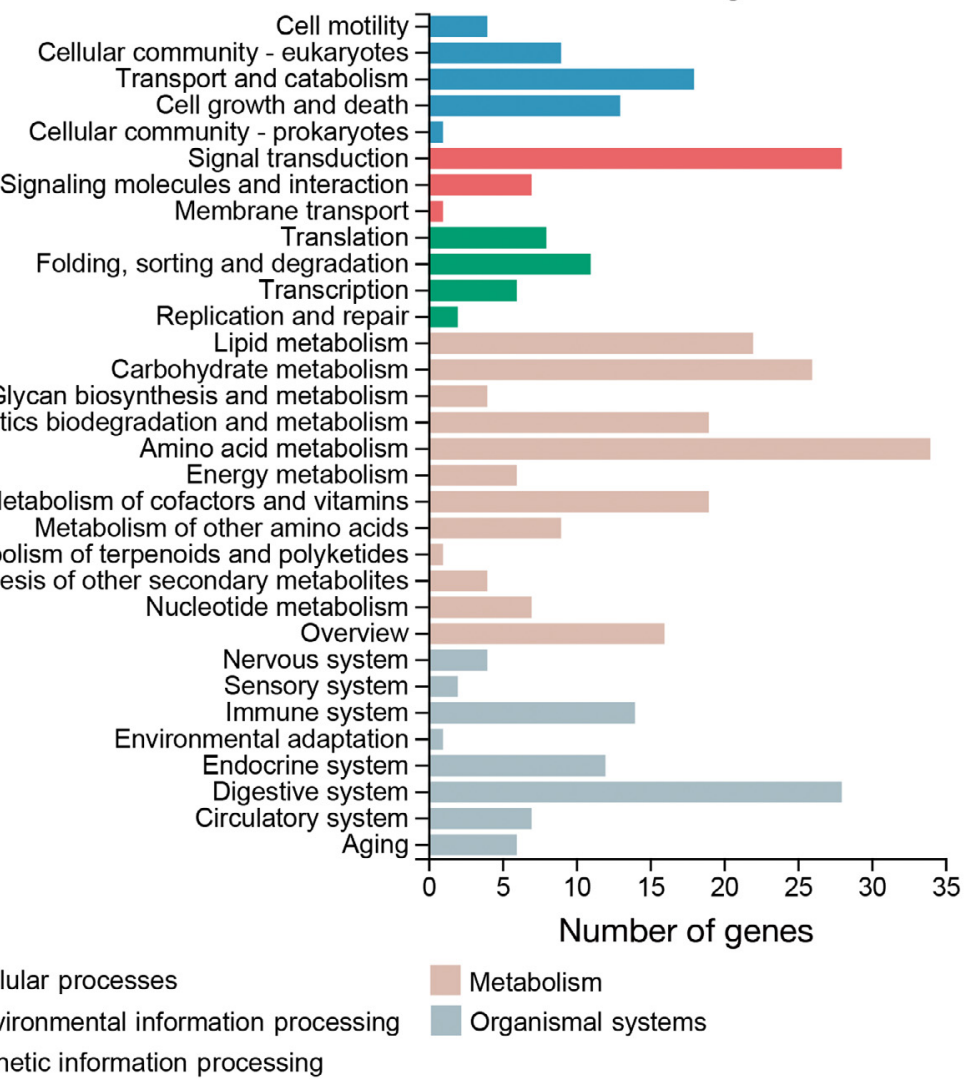

Fig. 5. KEGG pathway enrichment based on the differentially expressed genes (DEGs) of the yellow drum (A) 8unfed-vs.-16fed and (B)16unfed-vs. $-16 f e d$ groups. Horizontal axis shows the number of DEGs enriched in each pathway teins (Frick et al. 2008, McCue 2010). In our study, cytoplasm vacuolisation, blurred boundaries and cell dissolution (Fig. 2) were observed in the liver cells of individuals from the cold stress and starvation treatment groups due to mass reductions of the cells during starvation. In addition, compared to the control fish, the liver/body weight ratio in the 16unfed group was significantly lower, indicating consumption of liver inner mass during starvation. Interestingly, for fish in the low-temperature groups, where growth was inhibited, the liver/body weight ratios were not significantly different from those in the control group, suggesting mass consumption in both the liver and other parts of the body.

We conducted a transcriptomic analysis to examine the changes of gene expression profiles in the liver of yellow drum responding to low temperature and starvation. The low number of DEGs between the 8unfed and 8fed group indicated that the effect of coldinduced fasting in the 8fed group was similar to that in the 8unfed group. In the 8fed group, the fish stopped eating after the temperature reached $\sim 10^{\circ} \mathrm{C}$ on Day 7 , and starvation continued for another $15 \mathrm{~d}$ until the end of the experiment, which could explain their similar expression patterns on a transcriptomic level. In addition, the growth parameters and histological observations also showed a high resemblance between these 2 groups, suggesting that low temperature could be the main factor that affects fish during winter. In mammals, low temperature can affect the cellular pathways related to preventing protein denaturation and misaggregation, apoptosis, inhibition of transcription and translation, membrane permeability, etc. (van Breukelen \& Martin 2002). However, in our study, metabolism was one of the most enriched pathways during the cold and starvation stresses, e.g. the enzymes involved in carbohydrate metabolism, lipid metabolism and the digestive system were enriched in the 


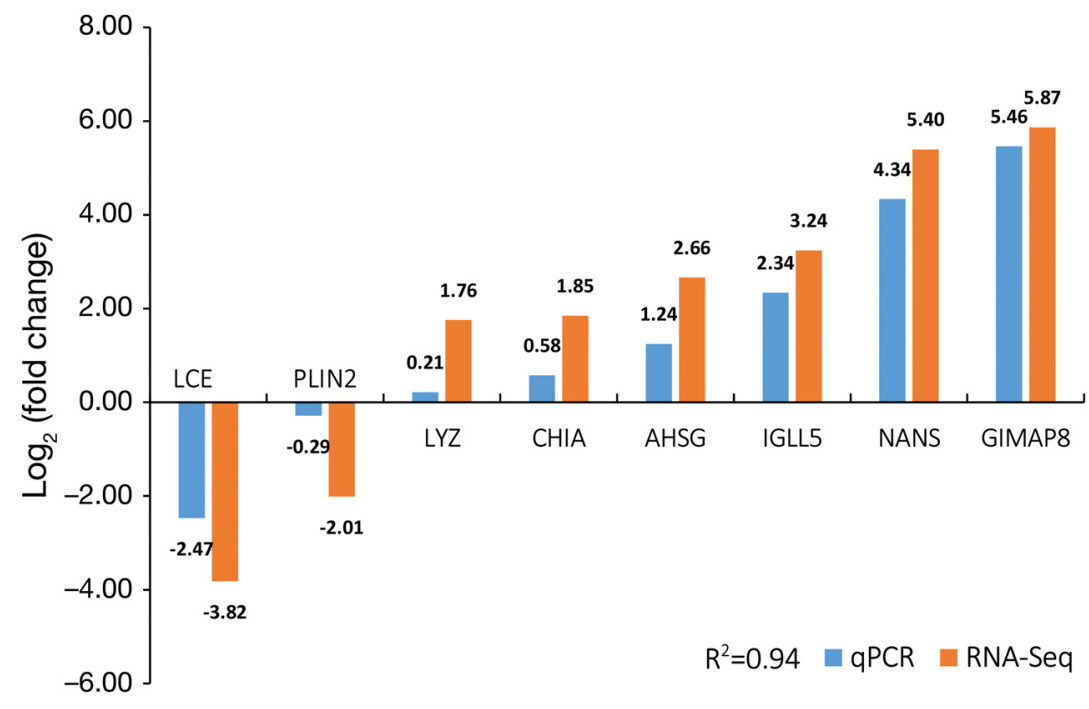

Fig. 6. Validation of yellow drum RNA-seq data by qPCR. Comparison of the relative $\log _{2}$ (fold change) values of 8 genes between RNA-seq and qPCR results immune-related genes in the 8unfedvs.-16fed group. Specifically, we identified 4 immune system genes which were DEGs specific to the 8unfedvs.-16fed group - including 2 coagulation factor $\mathrm{X}$ genes, 1 complement C3 gene and 1 soluble toll-like receptor 5 gene-and all of them were down-regulated in the 8fed group compared to the control. Since their important role in the immune system of fish and other animals has long been known, their down-regulation in the yellow drum under low temperature conditions might be a sign of an unsound immune system, which also explains occasional infections by opportunistic pathogens in winter.

\section{CONCLUSIONS}

the 8unfed-vs.-8fed group, the 16unfed-vs.-16fed group actually indicates the response of fish under starvation stress. Nevertheless, for most of the DEGs groups, including 8unfed-vs.-16fed, 16unfed-vs.16fed, 8fed-vs.-16fed and 8unfed-vs.-16unfed, the GO terms of 'metabolic process', 'cellular process', 'catalytic activity' and 'binding' contained the highest 4 numbers of DEGs. Similar results were also found in the KEGG enrichment, where the highest numbers of DEGs were involved in metabolism, signal transduction, etc. Hardewig et al. (1999) found that mitochondrial proliferation was a compensatory mechanism for the decelerating effect of low temperatures on metabolic processes, and in our study, energy-producing mitochondrial genes such as cytochrome oxidases were identified as the significant DEGs specific to the 8fed-vs.-16fed group, where the overexpression of cytochrome oxidase could be induced to produce more energy as needed. This is also consistent with the results found in a study of the emerald rockfish Trematomus bernacchii (Huth \& Place 2013).

Immunological suppression is likely another consequence of cold stress. The effect of temperature on the immune system in fish has been extensively investigated (Hurst 2007, Ibarz et al. 2010), where the integumental physical barrier (Ellis 2001, Micallef et al. 2012), humoral immune mediators (Fujita 2002) and inflammation signals (O'Shea \& Murray 2008) are often incorporated into immune responses. In our study, 61 DEGs annotated by KEGG and 15 DEGs with GO analysis were
Cold stress and starvation are commonly identified as the major drivers of overwinter mortalities in farmed fishes. To better understand the tolerance mechanisms underlying these stressors in marine fish, we investigated the effect of cold stress and starvation on the liver of yellow drum at histological and transcriptomic levels. Growth performance results showed both cold and starvation can cause growth inhibition; starvation caused mass loss in the liver and cold stress caused mass loss in both liver and other tissues. Histological observation revealed that cytoplasm vacuolisation, blurred boundaries and cell dissolution occurred in the liver cells of individuals in the cold and starvation treatment groups. Transcriptome analysis showed that genes related to metabolism were the most enriched DEGs during the low temperature and starvation stresses. These findings provide insights that can reveal the molecular mechanisms regulating the stress responses of yellow drum when exposed to low temperatures and starvation. This should help facilitate the establishment of management strategies during the overwinter stages to sustain the aquaculture industry.

Acknowledgements. This work was supported by grants from the National Natural Science Foundation of China (Nos. 41106114 and 31972785) as well as the Project of Zhejiang Province of China (2017C04003 and 2020C02015). The collection and handling of all animals used in this study was approved by the Animal Care and Use Committee of the Zhejiang Ocean University and the Marine Fisheries Re- 
search Institute of Zhejiang Province. All experimental procedures were performed in accordance to the prescribed guidelines.

\section{LITERATURE CITED}

Altschul SF, Gish W, Mille W, Myers EW, Lipman DJ (1990) Basic local alignment search tool. J Mol Biol 215:403-410

Anders S, Huber W (2013) Differential expression of RNASeq data at the gene level—the DESeq package. European Molecular Biology Laboratory, Heidelberg

Bilyk KT, Cheng CHC (2013) Model of gene expression in extreme cold-reference transcriptome for the highAntarctic cryopelagic notothenioid fish Pagothenia borchgrevinki. BMC Genomics 14:634

Boehlert GW, Yoklavich MM (1983) Effects of temperature, ration, and fish size on growth of juvenile black rockfish, Sebastes melanops. Environ Biol Fishes 8:17-28

Bolger AM, Lohse M, Usadel B (2014) Trimmomatic: a flexible trimmer for Illumina sequence data. Bioinformatics 30:2114-2120

Bruslé J, Anadon GG (1996) The structure and function of fish liver. Fish Morphol 76:545-551

* Donaldson MR, Cooke SJ, Patterson DA, Macdonald JS (2008) Cold shock and fish. J Fish Biol 73:1491-1530

Ellis AE (2001) Innate host defense mechanisms of fish against viruses and bacteria. Dev Comp Immunol 25:827-839

Favero G, Gimbo RY, Montoya LNF, Carneiro DJ, Urbinati EC (2020) A fasting period during grow-out make juvenile pacu (Piaractus mesopotamicus) leaner but does not impair growth. Aquaculture 524:735242

Frick NT, Bystriansky JS, Ip YK, Chew SF, Ballantyne JS (2008) Carbohydrate and amino acid metabolism in fasting and aestivating African lungfish (Protopterus dolloi). Comp Biochem Physiol A Mol Integr Physiol 151:85-92

Fujita T (2002) Evolution of the lectin-complement pathway and its role in innate immunity. Nat Rev Immunol 2: 346-353

Gallardo MA, Sala-Rabanal M, Ibarz A, Padros F, Blasco J, Fernandez-Borras J, Sanchez J (2003) Functional alterations associated with 'winter syndrome' in gilthead sea bream (Sparus aurata). Aquaculture 223:15-27

Hardewig I, Van Dijk PLM, Moyes CD, Portner HO (1999) Temperature-dependent expression of cytochrome- $C$ oxidase in Antarctic and temperate fish. Am J Physiol Regul Integr Comp Physiol 277:R508-R516

Hurst TP (2007) Causes and consequences of winter mortality in fishes. J Fish Biol 71:315-345

Huth TJ, Place SP (2013) De novo assembly and characterization of tissue specific transcriptomes in the emerald notothen, Trematomus bernacchii. BMC Genomics 14: 805 doi:10.1186/1471-2164-14-805

Hyvarinen P, Heinimaa S, Rita H (2004) Effects of abrupt cold shock on stress responses and recovery in brown trout exhausted by swimming. J Fish Biol 64:1015-1026

Ibarz A, Padros F, Gallardo MA, Fernandez-Borras J, Blasco J, Tort L (2010) Low-temperature challenges to gilthead sea bream culture: review of cold-induced alterations and 'winter syndrome'. Rev Fish Biol Fish 20:539-556

Kanehisa M, Araki M, Goto S, Hattori M and others (2008) KEGG for linking genomes to life and the environment. Nucleic Acids Res 36:D480-D484

Karasov WH, Pinshow B, Starck JM, Afik D (2004) Anatomical and histological changes in the alimentary tract of migrating blackcaps (Sylvia atricapilla): a comparison among fed, fasted, food-restricted, and refed birds. Physiol Biochem Zool 77:149-160

K Kirjasniemi M, Valtonen T (1997) Size-dependent over-winter mortality of young-of-the-year roach, Rutilus rutilus. Environ Biol Fishes 50:451-456

*Lamosova D, Macajova M, Zeman M (2004) Effects of shortterm fasting on selected physiological functions in adult male and female Japanese quail. Acta Vet Brno 73:9-16

KLangmead B, Salzberg SL (2012) Fast gapped-read alignment with Bowtie 2. Nat Methods 9:357-359

Livak KJ, Schmittgen TD (2001) Analysis of relative gene expression data using real-time quantitative PCR and the $2^{-\triangle \Delta C T}$ method. Methods 25:402-408

McCue MD (2010) Starvation physiology: reviewing the different strategies animals use to survive a common challenge. Comp Biochem Physiol A Mol Integr Physiol 156:1-18

* Micallef G, Bickerdike R, Reiff C, Fernandes JMO, Bowman AS, Martin SAM (2012) Exploring the transcriptome of Atlantic salmon (Salmo salar) skin, a major defense organ. Mar Biotechnol (NY) 14:559-569

* O'Shea JJ, Murray PJ (2008) Cytokine signaling modules in inflammatory responses. Immunity 28:477-487

* Pastoureaud A (1991) Influence of starvation at low-temperatures on utilization of energy reserves, appetite recovery and growth character in sea bass, Dicentrarchus labrax. Aquaculture 99:167-178

Koberts A, Pachter L (2013) Streaming fragment assignment for real-time analysis of sequencing experiments. Nat Methods 10:71-73

* Si YF, Wen HS, Li Y, He F, Li JF, Li SP, He HW (2018) Liver transcriptome analysis reveals extensive transcriptional plasticity during acclimation to low salinity in Cynoglossus semilaevis. BMC Genomics 19:464

Kong HB, Xu DD, Tian L, Chen RY, Wang LG, Tan P, You QC (2019) Overwinter mortality in yellow drum (Nibea albiflora): insights from growth and immune responses to cold and starvation stress. Fish Shellfish Immunol 92: 341-347

Thouzeau C, Robin JP, Le Maho Y, Handrich Y (1999) Body reserve dynamics and energetics of barn owls during fasting in the cold. J Comp Physiol B 169:612-620

* Trapnell C, Williams BA, Pertea G, Mortazavi A and others (2010) Transcript assembly and quantification by RNASeq reveals unannotated transcripts and isoform switching during cell differentiation. Nat Biotechnol 28:511-515

* van Breukelen F, Martin SL (2002) Invited review: molecular adaptations in mammalian hibernators: Unique adaptations or generalized responses? J Appl Physiol 92: 2640-2647

*Wang XL, Song Q, Wang ZY, Xie YJ, Zhang DL, Ye K, Han $F$ (2019) Characterizations of intracellular copper/zinc superoxide dismutase from yellow drum (Nibea albiflora, Richardson 1846) and its gene expressions under the ammonia/nitrite stress. Aquat Toxicol 214:105254

*Wen B, Jin SR, Chen ZZ, Gao JZ (2018) Physiological responses to cold stress in the gills of discus fish (Symphysodon aequifasciatus) revealed by conventional biochemical assays and GC-TOF-MS metabolomics. Sci Total Environ 640-641:1372-1381

*Wu X, Wang LG, Xie QP, Tan P (2020) Effects of dietary sodium butyrate on growth, diet conversion, body chemical compositions and distal intestinal health in yellow drum (Nibea albiflora, Richardson). Aquacult Res 51:69-79

Xu DD, Zhan W, Lou B, Mao GM, Shi HL, Xin J, Cheng GB (2010) Preliminary study on the growth characteristics 
of cage-cultured Nibea albiflora (Richardson). Fishery Modernization 37:34-37 (in Chinese with English abstract)

Xu DD, You QC, Chi CF, Luo SY, Song HB, Lou B, Takeuchi Y (2018) Transcriptional response to low temperature in the yellow drum (Nibea albiflora) and identification of genes related to cold stress. Comp Biochem Physiol Part D Genomics Proteomics 28:80-89

Xu H, Zhang DL, Yu DH, Lv CH, Luo HY, Wang ZY (2015) Molecular cloning and expression analysis of scd1 gene

Editorial responsibility: Per Gunnar Fjelldal,

Matredal, Norway from large yellow croaker Larimichthys crocea under cold stress. Gene 568:100-108

* Zhang XL, Li WY, Liu MH, Zhang X, Yin XL, Xu ZJ, Liu JH (2019) Transcriptomic analysis of the coldpretreated Larimichthys crocea showing enhanced growth fitness in cold water. Mar Biotechnol (NY) 21:791-805

* Zhu QH, Zhang LL, Li L, Que HY, Zhang GF (2016) Expression characterization of stress genes under high and low temperature stresses in the Pacific oyster, Crassostrea gigas. Mar Biotechnol 18:176-188

Submitted: December 10, 2019; Accepted: June 26, 2020 Proofs received from author(s): August 19, 2020 